Last-mile travel and bicycle sharing system in small/medium sized cities: user's preferences investigation using hybrid choice model Peer-reviewed author version

ADNAN, Muhammad; Altaf, S.; BELLEMANS, Tom; YASAR, Ansar \& Shakshuki, E. (2018) Last-mile travel and bicycle sharing system in small/medium sized cities: user's preferences investigation using hybrid choice model. In: Journal of Ambient Intelligence and Humanized Computing, 10(2), p.4721-4731..

DOI: $10.1007 / \mathrm{s} 12652-018-0849-5$

Handle: http://hdl.handle.net/1942/27273 


\title{
Last-mile Travel and Bicycle Sharing System in Small/Medium Sized Cities: User's Preferences Investigation using Hybrid Choice Model
}

\author{
Muhammad Adnan' \\ Corresponding Author, E-mail: muhammad.adnan@uhasselt.be \\ Office: +3211269147 \\ ORCID: 0000-0002-1386-2932
}

\author{
Shahbaz Altaf ${ }^{2}$ \\ E-mail: s0shah20@ cardmail.louisville.edu
}

Tom Bellemans ${ }^{1}$

E-mail: tom.bellemans@uhasselt.be

ORCID: 0000-0001-6839-3072

\section{Ansar-ul-Haque Yasar ${ }^{1}$ \\ E-mail: ansar.yasar@uhasselt.be \\ ORCID: 0000-0002-1542-2658}

\section{Elhadi M. Shakshuki ${ }^{3}$}

E-mail: elhadi.shakshuki@acadiau.ca

${ }^{1}$ UHasselt- Hasselt University, Transportation Research Institute (IMOB), Agoralaan, 3590 Diepenbeek, Belgium

${ }^{2}$ Department of Urban and Public Affairs, University of Louisville, Kentucky

${ }^{3}$ Jodrey School of Computer Science, Acadia University, Wolfville, Nova Scotia, Canada

\section{Abstract:}

First and last-mile access to and from public transport stations/stops is a major problem for encouraging public transport use. Bicycle sharing schemes have shown potential to fill this gap. Majority of studies examined the preference for using bicycle sharing schemes for large cities. Consequently, railway operators in the Netherland and Germany have started their own bike sharing schemes. Majority of the studies examined the preferences for using bike share schemes for larger cities. This study analyses the collected stated preference survey data for the use of bicycle sharing scheme for last mile travel, which is recently launched in small/medium sized cities of Belgium. Within this scheme a single docking station is available and users need to return bicycle at the same station. The survey also includes questions on respondents attitudes towards friendliness-to-cycling. The hybrid choice modelling framework is used to investigate preferences of users. Usual explanatory variables such as temperature, rain conditions, distance, rental cost, gender and age are found significant, which confirms the findings of earlier studies. Along with these; last-mile (to home) and an interaction term between rental cost and duration to keep bicycle are found significant, which indicate the negative effects of having a single docking station. Availability of escorting facility from parents/colleague/friends (a more common phenomenon in small/medium cities) also has a negative effect on the use of the examined bike share scheme. Paper also discusses promotional campaigns and marketing efforts according to the obtained results for making such schemes more successful.

\section{Keywords:}

Bicycle Sharing System; Last-Mile travel; Stated-Preference Survey; Hybrid Choice Model; Small/medium sized Cities in Belgium 


\section{Introduction}

A variety of mobility-related solutions is proposed to make cities smart, sustainable, congestion free and environment friendly. For example; electric mobility (Lützenberger et al. 2015), Carpooling (Knapen et al. 2014), efficient public transport scheduling (Avila-Torres et al. 2017), vehicle routing solutions (Wang et al. 2018), bicycling and bike sharing programs (Tang et al. 2011). The benefits of cycling at both individual and societal level are significant and well known. At an individual level, cycling provides a flexible and convenient way to complete short and medium length trips. Moreover, at the communal level, cycling assuage the problems like congestion, air pollution and global warming by substituting the trips made by cars. Therefore, cycling has been considered as a prime norm to facilitate sustainable transportation practices (Twaddle 2011). In order to promote cycling several approaches are being considered such as bicycling stimulation campaigns, provision of cycling related infrastructure and improving access for cyclist etc. (Pucher et al. 2010). However, a vastly popular method to promote and expand bicycling is the implementation of bicycling sharing schemes. These schemes can be seen in the cities and towns all across the world, which has helped in the development of the bicycling culture (BachandMarleau et al. 2012).

Public Bike Sharing Schemes (PBSS) have significant potential to resolve a major problem for promoting public transport (PT) i.e. the first and last-mile access. Therefore, efforts have been made to provide a better connection between the two systems such as placement of docking stations at or near major PT stations, coordination with the transportation schedules and an integrated payment system etc. Studies shown that integration of PBSS with PT can be complementary as overall trip travel time may reduce to six minutes (Jäppinen et al. 2013). However, for shorter trip lengths (less than $5 \mathrm{~km}$ ) PBSS can substitute PT (Bachand-Marleau et al. 2012).

The preferences of travellers for the use of bicycle were examined mainly by considering the availability of a bicycle to the user in the form of its ownership (Konstantinidou and Spyropoulou 2017; Wardman et al. 2007). Some studies investigate user preferences for the citywide infrastructure of PBSS installed in large cities (BachandMarleau et al. 2012; Feng and Li 2016), where PBSS infrastructure such as the number of bicycles and distance between the docking stations have been found as the major determinants of use of PBSS. However, limited studies exist that focused on determining the users preferences for use of PBSS for their inter-modal trip, especially with rail. One such recent study in this aspect is presented by Ji et al. (2017) for China, where they found that female, older and low-income rail commuters are less likely to use PBSS for accessing rail stations. Furthermore, rail commuters who have experienced bicycle theft and are travelling for mandatory activities such as work and school are likely to use PBSS.

This study focuses on investigating user's preferences for the use of PBSS in small/medium sized cities of Belgium (population in such cities are ranging from 30,000 to 200,000) where a single docking station exist which is usually located adjacent to the rail station. The prime aim of such PBSS is to facilitate rail commuters for their last-mile travel. However, to make PBSS successful in these cities it is required to study user's preferences in more detail because circumstances are different compared to large cities. For example in large cities; docking stations are available at a feasible distance and other travel alternatives are also available (such as high-frequency feeder bus service, taxi services such as Uber or regular types, car sharing and pooling options). User's preferences are examined by collecting information from a survey instrument that was designed using the standard stated 
preference approach. Additionally, with relevant socio-economic information, questions in relation to obtaining users attitude and opinions regarding their friendliness-to-cycling are also asked from the respondents. Collected data were analysed within the framework of discrete choice modelling using hybrid choice model. For comparison purposes, the traditional binary logit model was also estimated. The paper is structured as follows: Section 2 discusses literature review in detail which is followed by section 3 where discussion on the methodology adopted to design a survey instrument is presented. Section 4 explains the results obtained from the analysis of data followed by a detailed discussion on the results in section 5 . Section 6 concludes the paper.

\section{Literature Review}

In the last two decades, an overwhelming literature has been published on the bicycle use as a more sustainable solution of transport problems. As a result, a variety of approaches have been implemented in cities to promote this mode of travel, PBSS is one such approach. By the end of December 2017, it has been found that more than 1100 PBSS systems are operating in 63 countries of the world. As the focus of this paper is on the user's preferences for PBSS, therefore, the literature review presented here is focused on this aspect.

Most of the efforts in investigating users preferences and propensity for bicycle use have been done considering the availability of a bicycle to the user (i.e. users own a bicycle). Both revealed and stated preference data are being used in this regard. Wardman et al. (2007) examined the propensity of a cycle use for work trips in Great Britain and found that en-route facilities (such as segregated bikeways), the incentive to use cycle and tripend facilities (such as bike parking, shower facility etc) can improve the cycle use share. Buehler (2012) reported similar findings for a study in Washington D.C. region. Heinen et al. (2011) used factor analysis approach and found that decision to use a bicycle is mostly influenced by direct trip-based benefits (such as time savings and comfort etc), however, awareness (such as the effect of cycling on health and environment) influences frequent use and most importantly longer distance trips. Mode choice modelling studies are limited where user's attitude and opinions are incorporated using a more sophisticated and flexible model such as hybrid choice model (HCM) (Muñoz et al. 2016). Kamargianni and Polydoropoulou (2013) estimated an HCM to investigate effects of teenagers' attitude towards walking and cycling. They found that willingness to use active transport (walk and cycle) had the highest effect on the bicycle choice among teenagers.

In relation with PBSS, most of the studies utilized data available from the operators of bike share system (El-Assi et al. 2017), which can help relate ridership with surrounding and built environment variables. Kim et al. (2012) used the bike usage data from selected bike stations in a Korean city to investigate the role of station neighbourhood land use (such as residential and commercial), facilities (such as parks, schools and transit station) and the weather (such as rain). They found that in good weather conditions; commercial land use can cause 15 times more rides than residential areas, and parks cause 3 to 5 times more rides than transit stations and schools. A similar study (Claude 2014) for B-cycle a PBSS operating in Austin, Texas found that total feet of bicycle facility surrounding a PBSS station is positively correlated with levels of usage. Furthermore, the proximity of commercial centres was also a significant determinant of ridership levels. Similar findings were obtained in a study (Noland et al. 2016) for New York City (USA). Fishman et al. (2015) found that docking stations within 250 meters from the workplace and the age group representing 18-34 year's users are significant predictors of membership for PBSS. Another recent study from El-Assi et al. (2017) for PBSS in Toronto where station pair (OD) regression model was estimated, indicated a positive correlation between the increase in bicycle infrastructure and the decrease of 
the number of intersections at major roads. Results from their linear mixed effects model also indicated that temperature, station capacity, zonal population, employment density and station proximity to transit and universities were positively correlated with the usage of PBSS.

There exist a few notable studies that focused on user's preferences in the mode choice context for PBSS. Bachand-Marleau et al. (2012) reported that location of docking stations near the origins and destinations are the key to increase bike share usage. They also indicated that users who have experienced bicycle theft are more likely to use PBSS. A study (Yannis et al. 2015) for Athens (Greece), in which bike share was analysed as a competing mode with Car and PT indicated that travel time is the most important factor. Increase in the travel time is strongly deterring the choice of PBSS. Travel cost, comfort, age and gender also affect the choice for PBSS. A study (Feng and Li 2016) for Nanjing city (China) concluded that socio-economic factors (such as gender, employment status and car ownership) are more important than journey restriction variables in defining the user willingness to use PBSS. Another recent study (Campbell et al. 2016) used a stated preference data from Beijing (China) for determining user's preferences for conventional and electric bikes within a PBSS. They found that bike share demand is significantly negatively influenced by; trip distance, temperature, precipitation and poor air quality. They also found that demographic variables have no impact on the use of bike share. In relation with the electric bike, demographic variables were found important and negative influence was lesser for trip distance and temperature. However, precipitation has high significant negative influence. These studies have their focus on comprehensive PBSS implemented in the large cities and most of them analysed PBSS as a competing travel mode rather than a complementary mode.

User's preferences investigation considering the PBSS as a first/last-mile for multi-modal trips are scarce in the literature, despite the fact that PBSS have shown significant potential to complement mass PT systems. There exist a single study ( $\mathrm{Ji}$ et al. 2017) for a large city in China. The study found that demographic variables (Gender, Age, Income) are more important predictors of PBSS usage for access to rail stations. The significant reliance on demographic variables to determine PBSS usage raising equity concerns for investing in PBSS. Considering the knowledge gap, this study focuses on examining the users' preference for pro-rail type PBSS exist in small cities of many countries in Europe, with a single docking station located (in most of the cases) adjacent to transport hub or railway station. An example of such PBSS is the Blue-bike system in Belgium which is recently launched in small and medium cities. Furthermore, the analysis presented in this paper also incorporate attitude and opinions of users in HCM framework to get a better understanding of identifying potential groups for effective awareness and marketing strategies and campaigns.

\section{Methodology and Experiment Design}

\subsection{Modelling Framework}

HCM has been evolved to explicitly incorporate individual's attitude/perceptions with the goal of enhancing the behavioural representation of decision making in the choice modelling (Ben-Akiva et al. 2002a; Ben-Akiva et al. 2002b; Bolduc and Alvarez-Daziano 2010; Hess and Daly 2010). Walker (2002) mentioned that individual attitudes represent their taste, needs, values, goals and abilities that are evolved through experience and external factors, such as socio-economic characteristics. Within HCM setting, measurement model describes the 
relationship between indicators and an unobserved variable (often termed as the latent variable) and structural model explains the latent variable using observable characteristics of the individual.

In relation to this study, individual attitudes towards; environment preservation, their awareness regarding cycling as a healthy travel mode, their opinions about flexibility associated with the PBSS and restricting the use of motorized vehicle are considered using four indicators-type questions. These indicators are then related to the latent variable termed as friendliness-to-cycle (FC). Fig. 1 illustrates the modelling framework while mathematical formulation (following (Bolduc and Alvarez-Daziano 2010; Kamargianni and Polydoropoulou 2013)) is given through equations 1 to 4 . Explanatory variables $(X)$ such as rental cost, travel distance, weather conditions (temperature and rain), bicycling infrastructure (such as segregated lanes) along with the latent variable (FC) defines the utility of choosing a PBSS for access/egress to the rail station. The utility is measured by choice indicator (y). Additionally, explanatory variables in the form of individuals characteristics such as Age, Gender, driving license, car ownership and education status etc. are structurally explaining FC, which is measured via indicators. Equation 1 and 2 represent structural models as depicted through solid arrows in Fig. 1 and equation 3 and 4 represent measurements model depicted through a dashed arrow in Fig. 1.

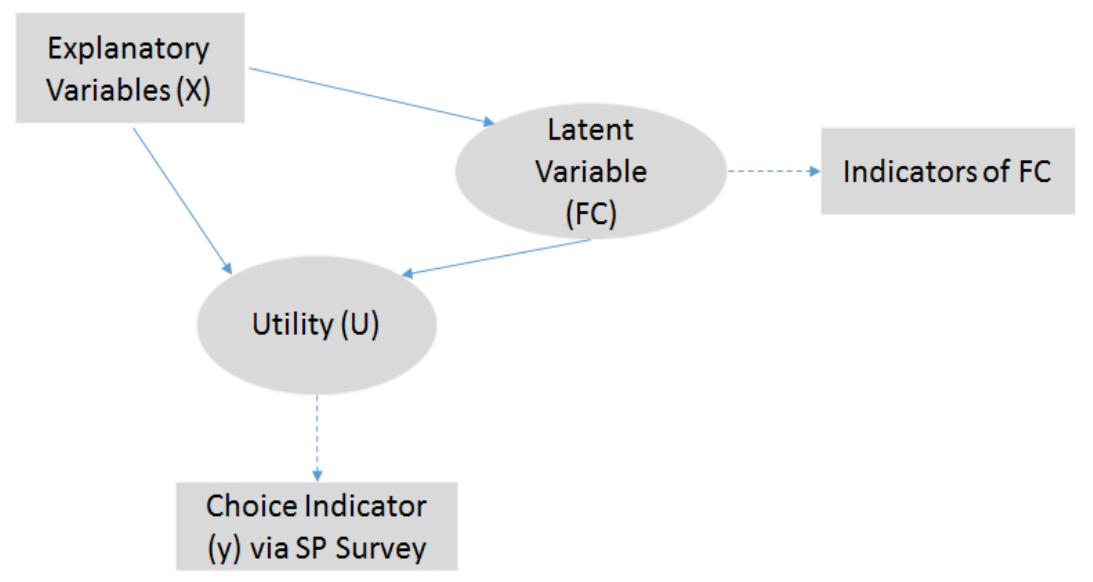

Fig. 1. Modelling framework (hybrid choice model)

$$
\begin{array}{lr}
F C=X_{1} \theta+\gamma & \gamma \sim N(0, \Sigma \gamma) \\
U_{b}=X_{2} \beta+\delta F C+\epsilon & \epsilon \sim N(0, \Sigma \epsilon) \\
I=\alpha+\lambda F C+\eta & \eta N(0, \Sigma \eta) \\
y=\left\{\begin{array}{lr}
1, \text { if } U_{b} \text { is maximum } \\
0, r & \text { otherwise }
\end{array}\right.
\end{array}
$$

where $X_{1}$ and $X_{2}$ are vectors of explanatory variables. $\theta$ is a vector of unknown parameters describing the effect of observable variables on FC. $\gamma$ represents a vector of random disturbance terms. $U_{b}$ represents utility of choosing a PBSS and $\beta$ represents a vector of estimated parameters of observed variables. $\delta$ is a diagonal matrix of unknown parameters associated with latent variable FC. $\epsilon$ is a vector of random disturbance terms associated with $U_{b}$. $I$ is a vector of four indicators of FC, $\alpha$ represents a vector of parameters that indicates the association between responses to scale and $\lambda$ is a vector of unknown parameters that provides the association of FC with indicators. $\eta$ 
is random error term and y is the choice indicator, having value 1 when PBSS is chosen and 0 otherwise. The likelihood function is given by

$L(y, I \mid X ; \Omega)=\int_{F C} P(y \mid X, F C ; \Sigma \epsilon) f_{I}(I \mid F C ; \lambda) f_{F C}(F C \mid X ; \eta) d_{F C}$

where $\Omega$ is the full set of parameters to estimate $(\theta, \lambda, \Sigma \epsilon)$ and $X$ is a vector of explanatory variables containing vectors $X_{1}$ and $X_{2}$. As the latent variable is known only to its distribution, therefore the joint probability of $\mathrm{y}, I$ and FC needs to be integrated over the latent constructs FC.

\subsection{Experimental Design}

This subsection discusses the design of the survey instrument and data collection methodology. A web-based survey questionnaire was developed which comprises of three major sections. In the first section, respondents socio-economic information (such as Gender, Age, Education, employment status, driving license, bicycle and car ownership, bicycle and other mode usages in relation with trip frequencies) was gathered. Additionally, awareness about PBSS, holding a membership for any PBSS and frequency and frequent trip purpose for the use of PBSS are also asked. The second section of the survey is comprised of a stated preference (SP) questionnaire, in which each respondent was given 9 different hypothetical scenarios for which he/she needs to indicate his/her choice in terms of using a PBSS or not for the last mile of their journey through the rail. More details of the SP questionnaire are given in the next paragraphs. In the third section, four key questions were asked from the respondents which indicate their attitude and perceptions towards their friendliness-to-cycling. The detailed discussion of the indicators and their association with latent variable terminologies can be found in (Muñoz et al. 2016). Respondents are required to indicate how much they agree/disagree with the following statements on a scale from 1 to 3 . Where, 1 = Agree, $2=$ Neutral, 3 = Disagree. The statement of these questions are as follows:

Q-3.1: Bicycle use helps improving air quality and overall environment.

Q-3.2: Use of bicycle helps in making me fit.

Q-3.3: Public bicycle sharing system is a more flexible and convenient option.

Q-3.4: Motorized transport should only be used for long distance trips (i.e. distance greater than $5 \mathrm{~km}$ ).

Selection of attributes for developing SP scenarios for section 2 of the questionnaire is based on a pilot survey that was conducted within Hasselt (a small city within Belgium). This pilot survey provided a list of attributes that are considered by the individuals for bicycle and PBSS use especially for the last-mile travel. Levels of the attributes are decided based on the available literature and also considering the operational characteristics of existing PBSS such as levels for the rental cost (which are mainly based on the duration for which bicycle has been taken for completing the journey). It is important to note that as in several small/medium cities there is only one docking station, so a user must need to return the bicycle to the same docking station. This situation entails that a user may eventually end up with keeping the bicycle for a longer period if the last mile travel is from the rail station to the home location or for an activity which required longer duration such as business-related activity. In our scenarios, we limited the level of this variable up to 48 hours (i.e. maximum duration to keep the bicycle), as the rental cost may increase exponentially after this time limit which is needed to maintain the availability of bicycle at the docking stations. Along with the rental cost, it is therefore, necessary to mention the duration. This 
is not an important issue in cases where PBSS are launched with several docking stations within the city (usually big cities). It is important to note that the modelling framework presented in the paper is generalized and can work for large cities. However, in large cities, there are multiple docking stations available in close proximity and therefore, the variables duration to keep the bicycle and bicycle parking at the destination, may not have such importance to include them in the choice experiments. Table 1 shows the list of attributes and their levels considered for different attributes for developing SP scenarios.

Table 1: SP Scenarios: Attributes and their levels

\begin{tabular}{ll}
\hline Attributes & Levels \\
\hline Bicycle rental cost (Euro) & Free, 1, 2, 3, 4, 5 \\
Duration to keep bicycle (hours) & $0.5,2,5,10,15,24,36,48$ \\
Trip Distance (Km) & Less than 1, 1 to 3, 3 to 5, more than 5 \\
Segregated bike lanes/paths & None, half pathway, Full pathway \\
Rainfall & Yes, No \\
Availability of other modes (for last mile travel) & Car-Escort, Bus, Taxi \\
Temperature (Celsius) & $0-5,5-15,15+$ \\
Bike Parking at Destination & Yes, No \\
\hline
\end{tabular}

Trip distance (for the last-mile travel), segregated bike lanes and temperature are those variables whose levels were designed from the available literature (Campbell et al. 2016; El-Assi et al. 2017; Fishman et al. 2015). Availability of other modes for the last-mile travel is considered because this could be a factor that may influence the choice of PBSS in a way that in small/medium cities bus routes are very limited, though taxi services are available at all times. Further, Escorting-Car (such as from parents/friends/Colleagues) is also found common for last-mile travel in such situation and therefore considered here as well. Bike parking at the destination is also considered important here because PBSS bicycles are generally good looking and mainly equipped with gearing systems, reflectors, bicycle baskets etc. These features on one hand increase attractiveness of PBSS among its users, however, on the other hand, it make the bicycle vulnerable to theft. As users may feel responsible for the security of the bike, therefore, in this context availability of bike parking at the destination is an important aspect. We considered only two levels for this variable to limit the generation of the number of SP scenarios. Overall, there are 8 attributes that need to be considered for the decision, which is suggested in many studies (Campbell et al. 2016; Kamargianni and Polydoropoulou 2013; Yap et al. 2016) as an optimal number of attributes that can be handled by the respondents. The further increase from this may result in more burden to the respondents.

In overall, 900 scenarios are generated and in the generation process, it is made sure that the main effects of the considered variables and some possible interactions such as between duration and rental cost are intact (i.e. partial factorial design is employed). Each respondent is presented with 9 scenarios in a randomized manner is a reasonable number as specified in the various relevant literature (Johnson and Orme 1996; Louviere et al. 2000). The scenarios presented to the users had two alternatives, choice for PBSS or not.

A pilot survey was conducted within Hasselt University in two official languages of Belgium (i.e. Dutch and French), to make sure respondents are able to understand the formulated questions and also able to perceive the SP scenarios as required. Their feedback was used to clarify various terms such as segregated bike lanes and the hypothetical scenario using real-life pictorial representations. Individuals were asked to consider a multi-stage trip via rail in small/medium cities (few city names are also provided as an example), in which presented scenario 
attributes are focusing on the last travel stage of such trip. Individuals were given two hypothetical situations with very clear instructions regarding consideration of a multi-stage rail trip, 1) last-mile to their home (i.e. last mile for home activity purpose, such multi-stage trips are return journeys from work) and 2) last-mile to some destination in any small/medium cities other than their residential city (i.e. last mile for activity other than home, such multi-stage trips are usually first leg of the journey for performing social/leisure or other activities). As an example, we provided a graphical illustration of such trip in the questionnaire for their readily understanding. These two hypothetical situations are equally randomized in the 9 profiles that have been assigned to one respondent (therefore, individual may have received either 5 scenarios based on the consideration of last-mile for home and 4 scenarios for last-mile for any other destination). Further, to keep the motivation and for high response rate, an incentive in the form of 20 euros was distributed among three respondents using a lucky draw. To distribute the survey questionnaire among the large audience, a variety of forums on social media such as Facebook and other platforms were contacted along with official channels within the Hasselt University. The questionnaire was distributed with a special focus on participants from small/medium cities within Belgium.

\section{Data and Model Estimation Results}

The data was collected mainly from the respondents living in over 30 small/medium sized cities of Belgium (i.e. having population between 30,000 to 200,000 residents). Majority of these responses are obtained from Hasselt, Sint-Truiden, Genk, Lier, Ostend, Aarshcot, Mons, Namur and Tongeren etc. Majority of these cities offer Public transport system in the form of buses within the city. The main connecting bus station is adjacent to the rail station, which is making that location as a transport hub. PBSS docking station is located nearby this transport hub. There exist few bus routes and the majority of them have a frequency of around 30 minutes to 1 hour. PBSS, therefore, can be attractive in such circumstances provided that it is available at reasonable cost for the longer duration. In total, responses from 321 respondents were considered complete and used for analysis after initial screening. These respondents have provided answers for the 9 randomly selected scenarios from the overall 900 generated scenarios, as explained in section 3. Descriptive statistics of the obtained data is presented in Table 2. It has been assured that the distribution of the respondents' demographic conditions are similar to overall Belgian demographic conditions so that the findings of the study can be generalized for all small/medium sized cities. The biggest differences are noted for age distribution, where from the survey only $3 \%$ of the respondents are from the age group of over 60 years. This is mainly because of the web-based nature of the methodology adopted to conduct this study. Earlier studies have indicated that older individuals have a significantly low tendency to use bike share system compared to use their own bicycle, therefore, lesser representation of such individuals in the survey is justifiable.

Table 2: Descriptive statistics

\begin{tabular}{|c|c|}
\hline Variable/Characteristics & Description and Statistics \\
\hline Gender & The survey participants were $59 \%$ Male and $41 \%$ Females \\
\hline Age & $\begin{array}{l}\text { Majority of the people }(57 \%) \text { who attempted the survey were between } 18-30 \text { years of age. } 40 \% \text { of the } \\
\text { respondent are falling in the age category of around } 31-60 \text { years. There were no responses from people } \\
<18 \text { years of age and a minute } 3 \% \text { of the responses are from the people }>60 \text { years old. }\end{array}$ \\
\hline Education Status & $\begin{array}{l}\text { The respondents mainly belonged to High School (24\%), Bachelors (15\%), Masters (48\%) and } \\
\text { Doctorate }(11 \%) \text { levels. The participation from other groups was negligible. }\end{array}$ \\
\hline Driving License & $\begin{array}{l}\text { Most of the people (79\%) had a driver license and substantial number (21\%) did not have a driving } \\
\text { license. }\end{array}$ \\
\hline Bicycle Ownership & $68 \%$ of the respondents owns a bicycle and around $32 \%$ in comparison did not have any cycle. \\
\hline Employment status & $\begin{array}{l}\text { Students }(31 \%) \text { and full-time employees ( } 48 \%) \text { dominated this category. In addition, Part-Time (11\\
%) } \\
\text { and Unemployed }(10 \%) \text { were also part of the mix. }\end{array}$ \\
\hline
\end{tabular}




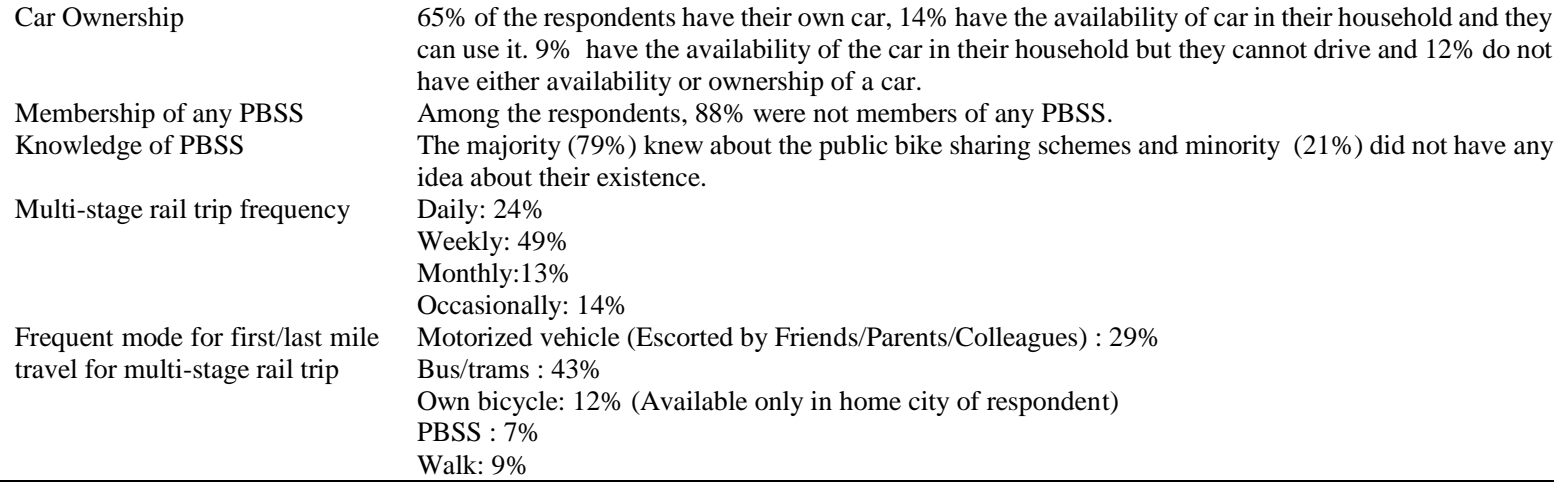
can use it. $9 \%$ have the availability of the car in their household but they cannot drive and $12 \%$ do not have either availability or ownership of a car.

Among the respondents, $88 \%$ were not members of any PBSS.

The majority (79\%) knew about the public bike sharing schemes and minority (21\%) did not have any idea about their existence.

Knowledge of PBSS

Daily: $24 \%$

Weekly: $49 \%$

Monthly: $13 \%$

Occasionally: $14 \%$

Frequent mode for first/last mile Motorized vehicle (Escorted by Friends/Parents/Colleagues) : 29\%

travel for multi-stage rail trip Bus/trams : $43 \%$

Own bicycle: $12 \%$ (Available only in home city of respondent)

PBSS : $7 \%$

Walk: $9 \%$

The question regarding household income was also asked, but the majority of the respondents choose not to answer that question. It is interesting to note that in table 2 that $75 \%$ of the respondents were highly educated (i.e. Degree holders). Along with holding of driving license and Car, they do possess their own bicycle as well. Most of them knew about PBSS but not holding its membership. It is also important to note that respondents multistage rail based trips are in the high-frequency range (i.e. daily and weekly), however, bus/tram are found to be their most frequent mode for last-mile travel of such trips. This situation further ascertaining that user preferences regarding PBSS are required to be studied in more detail. Variation in responses are also noted for all four indicators question for FC (see Fig. 2). High disagreement (48\%) is noted for Q:3.4, in which their opinion was sought about restricting the use of the motorized vehicle for longer distances.

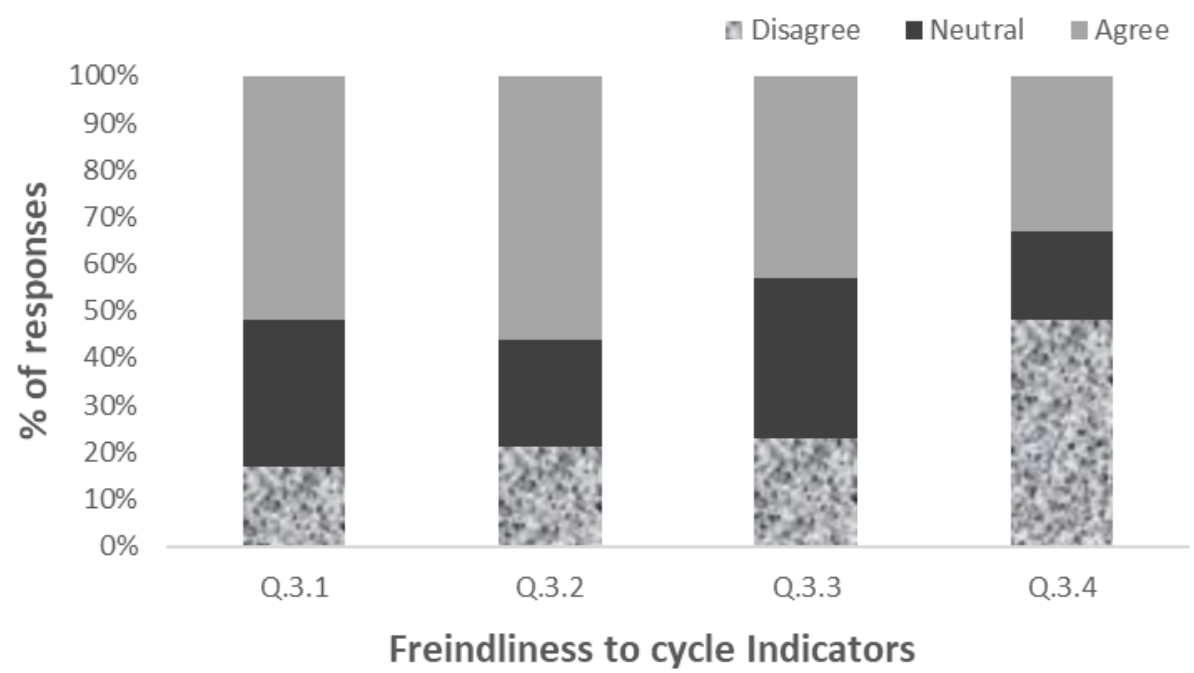

Fig. 2. Responses for Latent variable Indicators

The sample used for estimation of the model specified in the modelling framework consists of 2,889 observations from the SP related scenarios. $43.6 \%$ of observations have chosen PBSS as a mode for their last mile travel for multi-stage rail trip. Along with HCM model for comparison purpose a binary logit (BL) model was also estimated using the same number of observations. Both models were estimated using the Open Source Python Biogeme software (Bierlaire 2003; Bierlaire and Fetiarison 2009) and the number of draws for HCM model is used as 2000. The HCM model was estimated with an assumption that the latent variable FC increase the selection probability of PBSS. The structural equation that defines the relationship of FC with socio-economic 
characteristics is assumed as a linear regression equation. Table 3 present the model estimation results for BL and HCM. Further, Table 4 and 5 present the structural and measurement model estimation results specified using equations (1) and (3) respectively. Within HCM setting, parameters in equations (1) to (4) are jointly estimated using likelihood function specified as equation (5).

Table 3: Model estimation results for PBSS

\begin{tabular}{|c|c|c|c|c|}
\hline \multirow[t]{2}{*}{ Variables } & \multicolumn{2}{|c|}{ BL Model } & \multicolumn{2}{|c|}{ HCM Model } \\
\hline & Co-efficient & t-statistics & Co-efficient & t-statistics \\
\hline$\beta$ & 0.9239 & 1.1031 & -0.2651 & -2.5340 \\
\hline Bike Rental Cost (Euro) & -0.4368 & -9.8212 & -0.4312 & -10.243 \\
\hline $\ln ($ Duration (minutes) $)$ & NS & - & NS & - \\
\hline Bike Rental Cost (Euro) $* \ln ($ Duration (minutes) $)$ & -0.0060 & -10.560 & -0.0068 & -10.314 \\
\hline Trip Distance $(<1 \mathrm{Km})$ & -0.6707 & -20.349 & -0.6598 & -18.012 \\
\hline Trip Distance (1-3 Km) & 0.4775 & 13.121 & 0.4981 & 12.236 \\
\hline Trip Distance (3-5 Km) & 0.2357 & 12.356 & 0.2314 & 13.245 \\
\hline Availability of other Modes(Car-Escort) & -0.2891 & -4.1221 & -0.3181 & -5.2018 \\
\hline Availability of other Modes(Bus) & 0.3620 & 8.2314 & 0.3756 & 9.1945 \\
\hline Rainfall (Yes) & -1.2791 & -11.021 & -1.1342 & -12.315 \\
\hline Bike Parking at Destination (Yes) & 0.6945 & 2.0289 & 0.6867 & 2.3156 \\
\hline Segregated Bike Path (Full) & NS & - & NS & - \\
\hline Segregated Bike Path (half-way) & NS & - & NS & - \\
\hline Temperature $(0-5)$ & -0.3947 & -9.0438 & -0.3676 & -8.3176 \\
\hline Temperature (5-15) & 0.1987 & 3.3358 & 0.2154 & 4.3156 \\
\hline Last-mile (work to home leg of journey) & -0.0014 & 2.3526 & -0.0015 & 3.2564 \\
\hline FC & - & - & 0.9654 & 16.313 \\
\hline Adjusted $\rho^{2}$ & 0.2451 & & 0.2 & \\
\hline
\end{tabular}

Results in Table 3 shows BL and HCM parameters estimates that are found significant. As expected the HCM outperformed the BL model in terms of goodness of fit criteria (Adjusted $\rho^{2}$ ). This indicates that incorporation of the latent variable has increased the model explanatory power. Bike Rental Cost and duration to keep bicycle are considered continuous and all other variables are categorical. The variable duration (to keep the bicycle) is log-transformed to make the positive skew distribution as normal. Even with this transformation, this variable is not significant, however, the interaction term between bike rental cost and $\ln$ (duration) is significant. Trip distance, availability of other modes, temperature, rainfall and bicycle parking at destination are modelled using 4, 3, 3, 2 and 2 categories respectively and therefore for each of these variables 1 less parameter was estimated as the last category was taken as a base to avoid identification problem in the model estimation (de Dios Ortuzar and Willumsen 1994). All the estimated parameters seem to have reasonable signs, more importantly, the latent variable has the positive effect on the choice of PBSS.

Table 4: Structural Model estimation results for FC

\begin{tabular}{|c|c|c|}
\hline Structural & & \\
\hline Variables & Co-efficient & t-statistics \\
\hline$\theta_{F C}$ & 0.0512 & 9.1031 \\
\hline Gender (Male) & 0.1333 & 2.3212 \\
\hline Age (18-30) & 0.3652 & 4.3651 \\
\hline Age $(31-60)$ & 0.1197 & 3.5632 \\
\hline Employment (Student) & 0.2311 & 4.6121 \\
\hline Employment (Full-time) & -0.0411 & -3.1220 \\
\hline Education (High School) & NS & - \\
\hline Education (Bachelors) & NS & - \\
\hline Education (Masters) & NS & - \\
\hline Driving License (Yes) & NS & - \\
\hline Bike Ownership & 0.3966 & 4.0221 \\
\hline Car Ownership (Own Car) & NS & - \\
\hline Car Ownership (Available within a house) & NS & - \\
\hline Knowledge of PBSS (Yes) & NS & - \\
\hline
\end{tabular}


Table 5: Measurement Model estimation results

\begin{tabular}{lrr}
\hline Parameters & $\begin{array}{r}\text { Co- } \\
\text { efficient }\end{array}$ & t-statistics \\
\hline$\alpha_{1}$ & 0 & - \\
$\alpha_{2}$ & 0.4333 & 9.3212 \\
$\alpha_{3}$ & 0.1243 & 2.9871 \\
$\alpha_{4}$ & 0.5997 & 10.561 \\
$\lambda_{1}(\mathrm{Q}-3.1)$ & 1 & - \\
$\lambda_{2}(\mathrm{Q}-3.2)$ & 0.7109 & 13.128 \\
$\lambda_{3}(\mathrm{Q}-3.3)$ & 0.6251 & 14.127 \\
$\lambda_{4}(\mathrm{Q}-3.4)$ & 0.4934 & 2.981 \\
$\sigma_{1}$ & 1.3512 & 14.021 \\
$\sigma_{2}$ & 1.4523 & 10.724 \\
$\sigma_{3}$ & 1.4843 & 19.026 \\
$\sigma_{4}$ & 1.2318 & 3.4256 \\
\hline
\end{tabular}

Table 4 shows an estimation of the structural model for the latent variable. Variables included in the model are significant at the $95 \%$ significance level. The model results indicate that males are more likely to show friendliness to bicycling compared to females. Further, (18-30) years of age category is considering cycling more friendlier than higher age individuals. Students, as expected, showed more friendliness for cycling. Interestingly, education status and awareness regarding PBSS are found insignificant in explaining friendliness-to-cycling. Respondents having their own bike showed more friendliness to cycling. Respondents who used to complete their last-mile journey via Car(Escort) have shown less friendliness to cycling compared to the one who uses other modes such as buses and PBSS/Bicycle. In relation to table 5, where measurement model results are presented, the coefficient of the first indicator $\lambda_{1}$ was normalized to 1 . All parameters have come up with the expected sign. A more friendliness to cycling attitude will lead to respondents being more agreement with the statements about air quality improvement, their health and fitness, PBSS as a convenient option and allowing motorized transport only for long distance trips.

\section{Discussion}

The results of HCM showed that respondents attitude towards friendliness-to-cycling is important and significant and entail that unobservable characteristic should be implemented in the choice process to investigate users preferences for PBSS use. This has been found true in other mode choice modelling studies, and therefore, this study further confirms it. Among the variables, weather conditions i.e. rainfall and lower temperature negatively affect the choice of PBSS for the last mile. Similar findings are also presented in other studies (Campbell et al. 2016; El-Assi et al. 2017). It is interesting to see that segregated bike lanes availability is not significant, which is unlike many studies that advocated bicycle infrastructure as the main determinant for encouraging the use of bicycles (Kamargianni and Polydoropoulou 2013; Mateo-Babiano et al. 2016; Wardman et al. 2007; Yannis et al. 2015). The reason could be that in almost all cities of Belgium, availability of bicycle lanes/network is now a general norm and bicycle lanes are clearly demarcated for the encouragement of the bicycle use. Consistent 
investments in the bicycle infrastructure development and strict traffic regulations over the past few years within Belgium is therefore helped in reducing safety concerns among resident. In small/medium cities the safety concerns are much lesser due to the lower levels of traffic volume. Due to this situation during the choice experiments, respondents may have overlooked this variable while formulating their choice for the PBSS use. Availability of other modes in terms of an escorting-car (from parents/colleague/friends) reducing the choice of use of PBSS; as this is more comfortable, almost free of cost and may provide a socializing opportunity. Availability of bus increases the chance of use of PBSS, as in small/medium cities buses are available with low frequency, which means higher waiting time. PBSS is therefore, acting as a substitution mode when compared with buses for the last-mile travel, which is in-line with the finding of previous studies (Daddio and Mcdonald 2012). If the last-mile travel distance is within $1 \mathrm{~km}$, the use of PBSS will be lesser and individual may tend to prefer walking compared to renting a bike and take the responsibility for its security. It is interesting to note that parameter for distance (3-5km) is lower in comparison with (1-3km), signifying that distance up to $3 \mathrm{~km}$ is more acceptable to individuals and optimal cycling distance may lie somewhere between $3-5 \mathrm{~km}$.

As expected and in-line with previous studies, bicycle rental cost is found as an important deterrence factor for PBSS use (Buck et al. 2013; Wardman et al. 2007). It can be seen from the negative parameter of the interaction term for rental cost and duration that individuals do not want to keep bicycle for long period even when the rental cost is not increasing. However, the effect of this on the choice of PBSS is significantly lower compared to the rental cost. This suggest to believe that individuals are perceiving advantages of PBSS in terms of flexibility and convenience which comes at the cost of taking a responsibility to keep the bicycle secure as well until it is safely returned (to avoid the hassle and reporting of theft and damages even when there is no monetary penalty associated to it). This could be considered as the negative effect of having only one docking station in small and medium cities, as this situation cannot be encountered by the user of a PBSS which offer large number of docking stations (usually exist in large cities). The negative parameter of last-mile travel (for going home as part of return journey after performing work activity) also signifies this as home activity usually is conducted for long time period. Even though individuals can use the bike for the first-mile of the initial leg of a journey towards work on the next day and can return bicycle at that moment, however, it seems that individuals are perceiving this as an excessive binding/inconvenience to their freedom of choice not only for travel mode but for scheduling of other activities. This needs to be properly understood by the PBSS operators and promotion campaigns should also consider ways to increase the sense of security of bicycle among its users.

Installation of safe/secure bike parking facility at multiple locations within the city which doesn't mean complete return but taken away some responsibility from the user may enhance increase of PBSS use. For example, when user parked the bicycle at these parking facilities, they are no more burdened with reporting of bicycle theft and damages. However, users are still required to return bicycle to the main docking station. A smart solution could also be devised by developing a smartphone app and integrate it with operations of the PBSS. The app may notify the system user about multiple aspects such as reminding individual about bike return, generate alerts in case of theft and damages, display the location of the bike associated with the user during moving and parking conditions, availability of bike at the docking stations etc. Development of this infrastructure requires sufficiently lesser investments in comparison with developing docking stations with full facilities along with re-balancing operations. Such a smart solution can enhance the overall image and usability of the PBSS. On the other hand, it 
may increase the sense of security and reliability among the users and contribute towards raising the quality of life of its users (Chen and Chiu 2016). Furthermore, governmental agencies can also provide funding for such parking facilities as this can be used by other bicycle users and altogether bicycle usage may increase. Additionally, many PBSS operators are also launching electric bicycles which are expensive and are more vulnerable to theft. Consideration of such parking stations in small/medium sized cities is therefore more vital. The significance of variable, bike parking at the destination in the model also support the requirement of such intervention.

Students and individuals in the age group of 18-30 years have shown more cycle friendliness compared to full-time employees and individuals within the age group of 31-60 years. Similar findings were found by (Ji et al. 2017). Promotional campaigns therefore, can focus on these two groups. Offering incentives by contacting significant employers to offer free memberships or provide some corporate discounts can prove productive. Many institutes in Belgium provide some rewards to their employees who are regular bicycle users. PBSS operators can launch some mutual reward for employees of the institutes to use their service (i.e.PBSS) for the last-mile travel when they perform multi-stage rail trip. Additionally, it is noted that escorting-car (from parents/colleague and friends) reduces friendliness-to-cycling and also use of PBSS. Strict regulations on waiting and parking in surrounding regions of transport hub can curtail temptation regarding the use of such free of cost alternative.

\section{Conclusion}

Last-mile travel for inter-modal trips and use of PBSS in terms of users perspective is not studied in detail even for PBSS in larger cities. This study, however, focused on investigating preference of users within small/medium cities of Belgium where pro-rail PBSS has been launched to facilitate last-mile travel. Data was collected using web-based survey questionnaire from 30 such cities in the form of responses on SP scenarios, socio-economic characteristics, general travel habits and more importantly attitudes/perception on indicators of friendliness-tocycling. HCM a relatively advanced technique was used to investigate user preference in the choice context along with the incorporation of respondents attitude/perception (unobservable characteristics) in the selection of PBSS for their last-mile travel in multi-stage rail trip.

It has been concluded that HCM performs better in comparison with traditional BL model, suggesting that incorporation of unobservable characteristics improve the model explanatory power. Furthermore, it is noted that bike rental cost, inappropriate weather conditions, trip distance smaller than $1 \mathrm{~km}$ and availability of Escort-car (from parents/colleagues/friends) have negative influence on the choice of PBSS, however, availability of appropriate bike parking at destination and low frequency of buses improve the chances of PBSS selection. Additionally, the interaction term indicates that individuals are not interested to keep the PBSS's bicycle for longer periods even at a low rental cost. This is a special situation for PBSS in small/medium cities. It is suggested that safe/secure parking facilities where users of PBSS can give away some responsibility/burden of PBSS bicycle (i.e. the user will not require reporting if the bicycle is theft from such parking facility) enhance PBSS use. Additionally, PBSS operator should integrate the PBSS by developing a dedicated smartphone mobile app, which facilitate users and also operators on various aspects. Furthermore, attention should be paid towards designing incentives and promotional campaigns considering individuals who are a full-time employee and falling in the age group of 3144 years. 
Further research work involves improved survey design that considers individuals perceptions/attitudes in a much richer framework so that multiple specific latent variables can be deduced. Similar studies can also be done for PBSS in large cities. Additionally, a more comprehensive framework along with user preference is required to study the impacts of PBSS on increasing rail ridership, congestion reduction and other externalities.

\section{Acknowledgement}

We acknowledge the support of Ms. Nadine Smeyers and Mr. Marc Thoelen for translating the survey questionnaire in Dutch and French languages. The part of this work was presented in the $97^{\text {th }}$ Annual meeting of the Transportation Research Board, offering valuable reflections for during the publication of this study.

Conflict of interest: The authors declare that they have no competing interests.

\section{Compliance with ethical standards}

\section{References}

Avila-Torres P, Caballero R, Litvinchev I, Lopez-Irarragorri F, Vasant P (2017) The urban transport planning with uncertainty in demand and travel time: a comparison of two defuzzification methods Journal of Ambient Intelligence and Humanized Computing doi:10.1007/s12652-0170545-x

Bachand-Marleau J, Lee B, El-Geneidy A (2012) Better understanding of factors influencing likelihood of using shared bicycle systems and frequency of use Transportation Research Record: Journal of the Transportation Research Board:66-71

Ben-Akiva M et al. (2002a) Hybrid choice models: progress and challenges Marketing Letters 13:163175

Ben-Akiva M, Walker J, Bernardino AT, Gopinath DA, Morikawa T, Polydoropoulou A (2002b) Integration of choice and latent variable models Perpetual motion: Travel behaviour research opportunities and application challenges:431-470

Bierlaire M BIOGEME: a free package for the estimation of discrete choice models. In: Swiss Transport Research Conference, 2003. vol TRANSP-OR-CONF-2006-048.

Bierlaire M, Fetiarison M Estimation of discrete choice models: extending BIOGEME. In: Swiss Transport Research Conference (STRC), 2009.

Bolduc D, Alvarez-Daziano R On estimation of hybrid choice models. In: Choice Modelling: The Stateof-the-Art and the State-of-Practice: Proceedings from the Inaugural International Choice Modelling Conference, 2010. Emerald Group Publishing Limited, pp 259-287

Buck D, Buehler R, Happ P, Rawls B, Chung P, Borecki N (2013) Are bikeshare users different from regular cyclists? A first look at short-term users, annual members, and area cyclists in the Washington, DC, region Transportation Research Record: Journal of the Transportation Research Board:112-119

Buehler R (2012) Determinants of bicycle commuting in the Washington, DC region: The role of bicycle parking, cyclist showers, and free car parking at work Transportation research part D: Transport and Environment 17:525-531

Campbell AA, Cherry CR, Ryerson MS, Yang X (2016) Factors influencing the choice of shared bicycles and shared electric bikes in Beijing Transportation research part C: emerging technologies 67:399-414

Chen T, Chiu M-C (2016) Smart technologies for assisting the life quality of persons in a mobile environment: a review Journal of Ambient Intelligence and Humanized Computing doi:10.1007/s12652-016-0396-x

Claude C-M (2014) Factors that Affect Bicycle Ridership: A Case Study of the B-Cycle Bike Share System in Austin, Texas. University of Texas at Austin 
Daddio DW, Mcdonald N (2012) Maximizing bicycle sharing: an empirical analysis of capital bikeshare usage University of North Carolina at Chapel Hill

de Dios Ortuzar J, Willumsen LG (1994) Modelling transport. Wiley New Jersey,

El-Assi W, Mahmoud MS, Habib KN (2017) Effects of built environment and weather on bike sharing demand: a station level analysis of commercial bike sharing in Toronto Transportation 44:589613

Feng P, Li W (2016) Willingness to Use a Public Bicycle System: An Example in Nanjing City Journal of Public Transportation 19:6

Fishman E, Washington S, Haworth N, Watson A (2015) Factors influencing bike share membership: An analysis of Melbourne and Brisbane Transportation research part A: policy and practice 71:1730

Heinen E, Maat K, Van Wee B (2011) The role of attitudes toward characteristics of bicycle commuting on the choice to cycle to work over various distances Transportation research part D: transport and environment 16:102-109

Hess S, Daly A (2010) Choice Modelling: The State-of-the-art and the State-of-practice: Proceedings from the Inaugural International Choice Modelling Conference. Emerald Group Publishing Limited,

Jäppinen S, Toivonen T, Salonen M (2013) Modelling the potential effect of shared bicycles on public transport travel times in Greater Helsinki: An open data approach Applied Geography 43:1324

Ji Y, Fan Y, Ermagun A, Cao X, Wang W, Das K (2017) Public bicycle as a feeder mode to rail transit in China: The role of gender, age, income, trip purpose, and bicycle theft experience International Journal of Sustainable Transportation 11:308-317

Johnson RM, Orme BK How many questions should you ask in choice-based conjoint studies. In: Art Forum, Beaver Creek, 1996.

Kamargianni M, Polydoropoulou A (2013) Hybrid choice model to investigate effects of teenagers' attitudes toward walking and cycling on mode choice behavior Transportation Research Record: Journal of the Transportation Research Board:151-161

Kim D, Shin H, Im H, Park J Factors influencing travel behaviors in bikesharing. In: Transportation Research Board 91st Annual Meeting, 2012.

Knapen L et al. (2014) Exploiting graph-theoretic tools for matching in carpooling applications Journal of Ambient Intelligence and Humanized Computing 5:393-407 doi:10.1007/s12652-013-01974

Konstantinidou M, Spyropoulou I (2017) Factors affecting the propensity to cycle-the case of Thessaloniki Transportation Research Procedia 24:123-130

Louviere JJ, Hensher DA, Swait JD (2000) Stated choice methods: analysis and applications. Cambridge university press,

Lützenberger $\mathrm{M}$ et al. (2015) A common approach to intelligent energy and mobility services in a smart city environment Journal of Ambient Intelligence and Humanized Computing 6:337-350 doi:10.1007/s12652-015-0263-1

Mateo-Babiano I, Bean R, Corcoran J, Pojani D (2016) How does our natural and built environment affect the use of bicycle sharing? Transportation Research Part A: Policy and Practice 94:295307

Muñoz B, Monzon A, Daziano RA (2016) The Increasing Role of Latent Variables in Modelling Bicycle Mode Choice Transport Reviews 36:737-771

Noland RB, Smart MJ, Guo Z (2016) Bikeshare trip generation in New York city Transportation Research Part A: Policy and Practice 94:164-181

Pucher J, Dill J, Handy S (2010) Infrastructure, programs, and policies to increase bicycling: an international review Preventive medicine 50:S106-S125

Tang Y, Pan H, Shen Q Bike-sharing systems in Beijing, Shanghai, and Hangzhou and their impact on travel behavior. In: Transportation Research Board 90th Annual Meeting, 2011. vol 11-3862. 
Twaddle HA (2011) Stated preference survey design and pre-test for valuing influencing factors for bicycle use. Technische Universitat Munchen

Walker J (2002) Mixed logit (or logit kernel) model: Dispelling misconceptions of identification Transportation Research Record: Journal of the Transportation Research Board:86-98

Wang R, Zhou J, Yi X, Pantelous AA (2018) Solving the green-fuzzy vehicle routing problem using a revised hybrid intelligent algorithm Journal of Ambient Intelligence and Humanized Computing doi:10.1007/s12652-018-0703-9

Wardman M, Tight M, Page M (2007) Factors influencing the propensity to cycle to work Transportation Research Part A: Policy and Practice 41:339-350

Yannis G, Papantoniou P, Papadimitriou E, Tsolaki A Analysis of preferences for the use of a bicycling sharing system in Athens. In: International Cycling Safety Conference, 2015.

Yap MD, Correia G, Van Arem B (2016) Preferences of travellers for using automated vehicles as last mile public transport of multimodal train trips Transportation Research Part A: Policy and Practice 94:1-16 